\title{
The Non-invasive ${ }^{13} \mathrm{C}$-methionine Breath Test Detects Hepatic Mitochondrial Dysfunction as a Marker of Disease Activity in Non-AlCOHOLic Steatohepatitis
}

\author{
M. Banasch ${ }^{1}$, M.Ellrichmann ${ }^{1}$, A. Tannapfel ${ }^{2}$, W. E. Schmidt ${ }^{1}$, O. Goetze ${ }^{3}$ \\ ${ }^{1}$ Department of Medicine 1, St. Josef-Hospital, University of Bochum, ${ }^{2}$ Institute of Pathology, University of Bochum, Germany \\ ${ }^{3}$ Division of Gastroenterology and Hepatology, University Hospital Zurich, Switzerland
}

\begin{abstract}
Introduction: Mitochondrial dysfunction plays a central role in the general pathogenesis of non-alcoholic fatty liver disease (NAFLD), increasing the risk of developing steatosis and subsequent hepatocellular inflammation. We aimed to assess hepatic mitochondrial function by a non-invasive ${ }^{13} \mathrm{C}$-methionine breath test (MeBT) in patients with histologically proven NAFLD.

Methods: 118 NAFLD-patients and 18 healthy controls were examined by MeBT. Liver biopsy specimens were evaluated according to the NASH scoring system.

Results: Higher grades of NASH activity and fibrosis were independently associated with a significant decrease in cumulative ${ }^{13} \mathrm{C}$-exhalation (expressed as cPDR(\%)). cPDR $1.5 \mathrm{~h}$ was markedly declined in patients with NASH and NASH cirrhosis compared to patients with simple steatosis or borderline diagnosis (cPDR1.5h: $3.24 \pm 1.12 \%$ and $1.32 \pm 0.94 \%$ vs. $6.36 \pm$ $0.56 \%$ and $4.80 \pm 0.88 \%$ respectively; $\mathrm{p}<0.001) .{ }^{13} \mathrm{C}-$ exhalation further declined in the presence of advanced fibrosis which was correlated with NASH activity $(r=0.36)$. The area under the ROC curve (AUROC) for NASH diagnosis was estimated to be 0.87 in the total cohort and 0.83 in patients with no or mild fibrosis (F0-1).

Conclusion: The ${ }^{13} \mathrm{C}$-methionine breath test indicates mitochondrial dysfunction in non-alcoholic fatty liver disease and predicts higher stages of disease activity. It may, therefore, be a valuable diagnostic addition for longitudinal monitoring of hepatic (mitochondrial) function in non-alcoholic fatty liver disease.
\end{abstract}

Key words: ${ }^{13} \mathrm{C}$-methionine breath test, MeBT, NASH

\section{INTRODUCTION}

Non-alcoholic fatty liver disease (NAFLD) is currently the most common liver disease in the developed world and has been recognized as a leading cause of cryptogenic liver cirrhosis.

The pathogenesis of NAFLD has been tightly linked to insulin resistance (IR) and the metabolic syndrome, although the progression from steatosis to steatohepatitis (NASH) is not well understood [1-3].

Accumulating evidence indicates that mitochondrial dysfunction plays a central role in the pathogenesis of NAFLD both for steatosis development and subsequent hepatocellular inflammation, raising the possibility that NAFLD is a mitochondrial disease [4]. Accordingly, morphologic changes in liver mitochondria and evidence of oxidative stress have been observed in patients and animal models with NASH. These mitochondrial abnormalities include depletion of mitochondrial ( $\mathrm{mtDNA}$ ), decreased activity of respiratory chain complexes and impaired $\beta$-oxidation [5-9].

While decreased oxidation of fatty acids favours the development of steatosis, respiratory chain dysfunction can directly lead to the production of reactive oxygen species (ROS), resulting in lipid peroxidation and initiation of mitochondrial apoptotic pathways.

Liver biopsy may be essential for primary diagnosis of NASH as it represents the only diagnostic procedure that can distinguish (benign) steatosis from steatohepatitis, and allows accurate evaluation of fibrosis, but due to its invasive character it is ineligible for frequent monitoring of disease progression or surveillance of treatment interventions.

The ${ }^{13} \mathrm{C}$-methionine breath test $(\mathrm{MeBT})$ is a non-invasive diagnostic instrument for in vivo assessment of hepatic mitochondrial function. Methionine is an important donor of methyl groups and mainly metabolized by hepatic transmethylation, resulting in the production of a carbon fragment at the oxidation level of formaldehyde which can be finally converted into $\mathrm{CO}_{2}$. A key enzyme of this metabolic pathway, the sarcosine oxidase complex, seems to be present exclusively in the mitochondria. Therefore the proportion of ${ }^{13} \mathrm{CO}_{2}$ produced from orally administered methyl${ }^{13} \mathrm{C}$ labelled methionine could serve as an indicator of hepatic mitochondrial oxidation capacity.

In recent clinical trials, the MeBT has been used to explore mitochondriotoxic effects of alcohol, drug induced steatohepatitis and, in particular, chronic HIVinfection [10-12]. Although the proportion of exhalated ${ }^{13} \mathrm{CO}_{2}$ is rather small $(6-8 \%)$ even in healthy subjects, we could clearly separate conditions of hepatic mitochondrial stress with an excellent inter- and intraindividual reproducibility [13-15]. More recently, we have demonstrated significant improvement of hepatic mitochondrial function in a patient with non-alcoholic steatohepatitis and metabolic syndrome after 
treatment with the CB1-receptor blocker rimonabant [16].

Other breath test substrates, such as methacetin or caffeine which are metabolised by microsomal cytochrome P450 enzymes, have been used for monitoring general hepatic function in NAFLD. These studies have demonstrated decreased microsomal activity in patients with NASH and advanced fibrosis. However, a substrate metabolised by mitochondrial decarboxylation might be more sensitive and, in particular, specific for detecting mitochondrial dysfunction in early pre-fibrotic stages of NASH $[17,18]$. The present study was, therefore, aimed (i) to investigate hepatic mitochondrial function by MeBT in metabolically well characterised patients with NASH, and (ii) to identify potential histological or biochemical parameters correlating with individual breath test outcome.

\section{METHODS}

This cross-sectional study was carried out according to Good Clinical Practice and the Declaration of Helsinki. Written informed consent was obtained from all participants and the local Ethics Committee approved the protocol. 118 patients with suspected non-alcoholic fatty liver (NAFL) underwent histological examination of the liver after exclusion of other causes of steatotic liver disease, such as viral hepatitis $B$ or $C$ viral infection, autoimmune or inherited liver disease and toxic hepatitis (alcohol or drug induced). 18 lean healthy individuals without hepatic steatosis (assessed by ultrasound) and normal liver laboratory values served as controls. Detailed characteristics of patients and controls are given in Table 1.

\section{LIVER BIOPSY}

The indication for performing a liver biopsy was based purely on clinical reasons. Liver tissue samples were obtained by laparoscopic or ultrasound guided percutaneous needle biopsy within four weeks before study entry, and evaluated according to the NASH scoring system for steatosis, lobular inflammation, liver cell injury, and fibrosis [19]. The NASH activity score (NAS) was calculated by adding individual scores of steatosis, lobular inflammation and ballooning, and ranked from 3-8. Patients were subdivided into four histological groups: simple steatosis (NAS $<3$ ), borderline diagnosis (NAS 3-4), definite NASH (NAS 5-8), and NASH cirrhosis.

\section{${ }^{13}$ C-Methionine Breath Test (MeBT)}

The detailed test procedure is described elsewhere [13]. Briefly, each patient received $2 \mathrm{mg} / \mathrm{kg}$ body weight (methyl-13 C)-labelled methionine (99\% atom isotopic enrichment, Cambridge Isotope, Andover, MA, USA) dissolved in $100 \mathrm{ml}$ water. Breath samples were obtained before substrate administration and at 10 minute intervals for 90 minutes. The ${ }^{13} \mathrm{C} /{ }^{12} \mathrm{C}$ isotope ratio of the breath samples was analysed by nondispersive isotope selective infrared spectroscopy (IRIS, Wagner Analysen Technik, Bremen, Germany). Primary results were expressed as the delta $(\delta){ }^{13} \mathrm{C} /{ }^{12} \mathrm{C}$ isotope ratio over baseline (DOB). To measure the proportion of the metabolized substrate, the results were expressed as percentage dose of ${ }^{13} \mathrm{C}$ recovered (PDR) over time for each time interval and cumulative PDR (cPDR $\left.{ }_{1.5 \mathrm{~h}}\right)$ after 90 min test time.

Table 1. Baseline data and histological findings of 118 patients with histologically proven non-alcoholic fatty liver disease (NAFLD) and 18 lean controls. Data is presented as mean (SD).

\begin{tabular}{|c|c|c|c|c|c|}
\hline Variable & $\begin{array}{c}\text { Lean } \\
\text { Controls } \\
\mathbf{n}=18\end{array}$ & $\begin{array}{c}\text { Simple } \\
\text { Steatosis } \\
\mathbf{n}=11\end{array}$ & $\begin{array}{c}\text { Borderline } \\
\text { NASH } \\
n=47\end{array}$ & $\begin{array}{c}\text { Definite } \\
\text { NASH } \\
n=53\end{array}$ & $\begin{array}{c}\text { NASH } \\
\text { Cirrhosis } \\
\mathbf{n}=7\end{array}$ \\
\hline \multicolumn{6}{|l|}{ Gender } \\
\hline Male, $\mathrm{n}$ & 11 & 7 & 31 & 36 & 5 \\
\hline Female, $\mathrm{n}$ & 7 & 4 & 16 & 17 & 2 \\
\hline Age, yr & $44.0(10)$ & $51.0(12)$ & $50.5(12)$ & $49.5(12)$ & $59.0(6)$ \\
\hline BMI, $\mathrm{kg} / \mathrm{m}^{2}$ & $24.4(2.3)$ & $24.6(4.6)$ & $27.4(7.2)$ & $30.3(6.3)^{\mathrm{a}}$ & $31.1(4.6)^{\mathrm{a}}$ \\
\hline Serum ALT, U/1 & $26(8)$ & $41(12)$ & $67(40)^{a}$ & $94(64){ }^{a} a$ & $64(30)^{a}$ \\
\hline Cholesterol, mg/dl & $172(12)$ & $243(39)$ & $235(47)$ & $216(49)$ & $189(48)$ \\
\hline Triglycerides, mg/dl & $136(17)$ & $142(75)$ & $239(1172)$ & $211(127)$ & $185(118)$ \\
\hline Impaired glucose tolerance, $n$ & 0 & 0 & 15 & 21 & 2 \\
\hline Diabetes mellitus Type 2, n & 0 & 2 & 9 & 5 & 3 \\
\hline \multicolumn{6}{|l|}{ Fibrosis score, $\mathrm{n}$} \\
\hline F 0 & - & 9 & 11 & 2 & - \\
\hline F 1 & - & 1 & 29 & 28 & - \\
\hline F 2 & - & 1 & 3 & 10 & - \\
\hline F 3 & - & 0 & 4 & 13 & - \\
\hline
\end{tabular}

${ }^{a} \mathrm{p}<0.01$ vs. simple steatosis and lean controls by Dunn's post hoc test

Abbreviations: ALT: alanine amino-transferase; BMI: body mass index; n: number of patients 


\section{Biochemical Measurements}

Biochemical evaluations included alanine aminotransferase (ALT), total cholesterol, triglycerides, and an oral glucose tolerance test (OGTT). Impaired or diabetic glucose tolerance was defined by the criteria of the American Diabetes Association.

\section{STATISTICS}

Statistical analysis was firstly carried out as a descriptive evaluation of cPDR1.5h (\%) and clinical characteristics of the patients. All data is presented as mean $\pm \mathrm{SD}$, unless otherwise specified. Differences in hepatic mitochondrial function, as assessed by MeBT, of different histological groups were tested by KruskalWallis ANOVA and Dunn's post hoc tests. The relationship between categorical variables of liver histology (NASH activity and fibrosis) and breath test outcome was analysed by correlation analyses. The diagnostic performance of $\mathrm{cPDR}_{1.5 \mathrm{~h}}$ to detect different histological features was assessed by using receiver operating characteristic (ROC) curves, and the area under the ROC curve (AUROC) was used as a parameter of accuracy. Optimal cut-off values for MeBT were chosen to obtain suitable sensitivity and specificity for clinical decision making. To define the relationship between MeBT results as expressed by $\mathrm{cPDR}_{1.5 \mathrm{~h}}$ and a set of surrogate observations of the patient group, a multiple linear regression model using the procedure for general linear models with $\mathrm{cPDR}_{1.5 \mathrm{~h}}$ as the dependent variable and a set of explanatory variables (BMI, ALT, cholesterol, triglycerides, and diabetic predisposition) was applied. In this model, all covariates have been included based on an a priori decision guided by scientific knowledge and biologic plausibility. The results were regarded as significant when the error probability was less than 0.05. Statistical analysis and graphics were carried out by commercial software programs (Graph PAD Prism, version 4.01, San Diego, CA).

\section{RESULTS}

\section{Patient Characteristics and Histopathology RESULTS}

All measurements were completed without complications or adverse events. Liver histology confirmed the presence of NAFLD in all cases. The size of biopsy specimens ranged from 18 to $32 \mathrm{~mm}$ and was considered adequate for the evaluation with the scoring systems employed by the pathologist. The major clinical, biochemical and histological parameters of the participants included in the analysis are listed in Table 1.

11 patients presented with simple steatosis, 47 cases were diagnosed as borderline and 53 as definite NASH. The majority of patients $(n=81)$ presented with no or mild fibrosis (F0-1), and only 7 patients had complete cirrhotic conversion. Impaired glucose tolerance (IGT) or type-2 diabetes was diagnosed in $49 \%$ of patients with predominance within the "borderline" and NASH group. ALT and BMI were significantly higher in patients with definite or borderline NASH and NASH cirrhosis compared to individuals with simple steatosis or lean controls (Table 1).
Relationship between BReath Test Outcome And Different Histological Groups

Cumulative ${ }^{13} \mathrm{C}$-Exhalation $\left(\mathrm{cPDR}_{1.5 \mathrm{~h}}\right.$ ) ranged from 0.2 to $6.9 \%$ and was lower in patients with definite NASH $(3.24 \pm 1.12 \%)$ and NASH cirrhosis $(1.32 \pm$ $0.94 \%)$ compared to individuals with simple steatosis $(6.36 \pm 0.56 \%)$ or borderline diagnosis $(4.80 \pm 0.88 \%$; each $\mathrm{p}<0.001)$. Lean controls $\left(\mathrm{cPDR}_{1.5 \mathrm{~h}}: 6.15 \pm 1.2 \%\right)$ and patients with steatosis or borderline diagnosis could not be separated by MeBT, as illustrated by box plot analyses (Fig. 1).

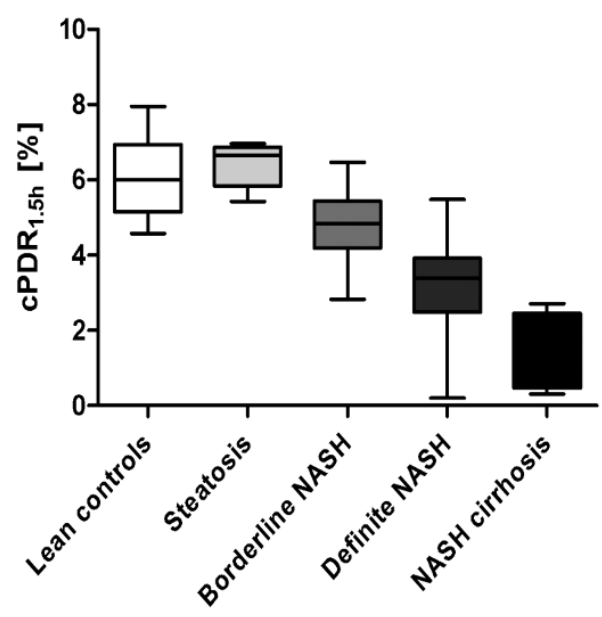

Fig. 1. Cumulative ${ }^{13} \mathrm{C}$-exhalation after $1.5 \mathrm{~h}$ test time $\left(\mathrm{cPDR}_{1.5 \mathrm{~h}}\right)$ in 118 patients with non-alcoholic fatty liver disease (NAFL) according to different histological stages (simple steatosis, borderline diagnosis, definite NASH, and NASH cirrhosis) and 18 lean controls.

$\mathrm{p}<0.001$ for definite NASH and NASH cirrhosis vs. lean controls, simple steatosis and borderline diagnosis by Kruskal-Wallis ANOVA and Dunn's post hoc tests.

To further explore the influence of fibrosis on hepatic methionine metabolism, additional analyses in subgroups of patients with minimal (F0-1) and advanced (F2-3) fibrosis stages were performed (Fig. 2). Advanced fibrosis (F2-3) was associated with a significant decline in ${ }^{13} \mathrm{C}$-exhalation both in the borderline and NASH subgroup $\left(\mathrm{cPDR}_{1.5 \mathrm{~h}}: 4.99 \pm 0.71 \%(\mathrm{~F} 0-1)\right.$ vs. $2.88 \pm 0.78 \%(\mathrm{~F} 2-3)$ in borderline NASH, and 3.82 $\pm 0.87 \%$ (F0-1) vs. $2.50 \pm 0.95 \%$ (F2-3) in definite NASH; $\mathrm{p}<0.001$ and $\mathrm{p}<0.05$, respectively).

In patients with mild fibrosis, the difference in $\mathrm{cPDR}_{1.5 \mathrm{~h}}$ between borderline and definite NASH remained significant $\left(\mathrm{cPDR}_{1.5 \mathrm{~h}}: 4.99 \pm 0.71 \%\right.$ vs. $3.82 \pm$ $0.87 \% ; \mathrm{p}<0.001$ ), but in patients with advanced fibrosis, it was not possible to discriminate between borderline and definite NASH or cirrhosis $(2.88 \pm 0.78 \%$ vs. $2.50 \pm 0.95 \%$ vs. $1.32 \pm 0.94 \%$; each $\mathrm{p}=$ n.s.) $($ Fig. 2$)$. Correlation analyses confirmed the synergistic negative effect of NASH activity and fibrosis on individual breath test outcome $(r=-0.62$ for NAS and $r=-0.77$ for fibrosis stage; each $\mathrm{p}<0.001)$. NASH activity and fibrosis were correlated $(\mathrm{r}=0.36, \mathrm{p}<0.001)$.

The relationship between histological parameters, selected biochemical variables (Chol, Trig, ALT, and 


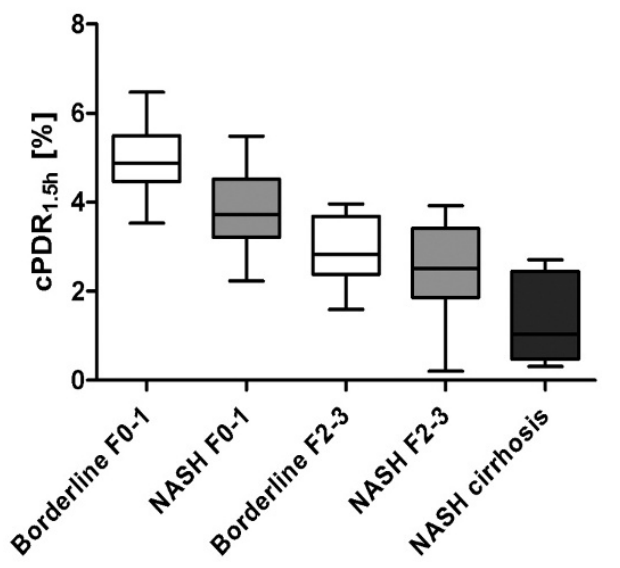

Fig. 2. Cumulative ${ }^{13} \mathrm{C}$-exhalation after $1.5 \mathrm{~h}$ test time $\left(\mathrm{PDR}_{1.5 \mathrm{~h}}\right)$ in 99 patients with borderline diagnosis or definite NASH stratified for the presence of advanced (F2-3) fibrosis and 7 patients with complete cirrhotic conversion.

$\mathrm{p}<0.001$ for F0-1 vs. F2-3 in each group and for F0-1 vs. cirrhosis;

$\mathrm{p}<0.05$ for $\mathrm{F} 0-1$ with borderline diagnosis vs. F0-1 with definite NASH

$\mathrm{p}=$ n.s. for F2-3 vs. cirrhosis by Kruskal-W allis ANOVA and Dunn's post hoc tests.

diabetic predisposition) and breath test outcome was further studied by multivariate analysis (Table 2). The majority of variance $(71 \%)$ could be attributed to histological parameters. Cholesterol was the only biochemical variable with a significant and positive association with $\mathrm{cPDR}_{1.5 \mathrm{~h}}(\mathrm{r}=0.39, \mathrm{p}<0.001)$ but could explain only $3.3 \%$ of variance in the entire model.

\section{ReCEIVER-OPERATING CHARACTERISTIC (ROC) CuRve ANALYSIS OF MeBT PARAMETERS}

ROC curves of cumulative ${ }^{13} \mathrm{C}$-excretion $\left(\mathrm{cPDR}_{1.5 \mathrm{~h}}\right)$ were plotted to describe the diagnostic performance of MeBT for the discrimination between patients with definite NASH and those with only borderline diagnosis or simple steatosis (Fig. 3a). The area under the ROC curve (AUROC) was 0.87 for all fibrosis stages and 0.83 for patients with only mild fibrosis (F0-1).

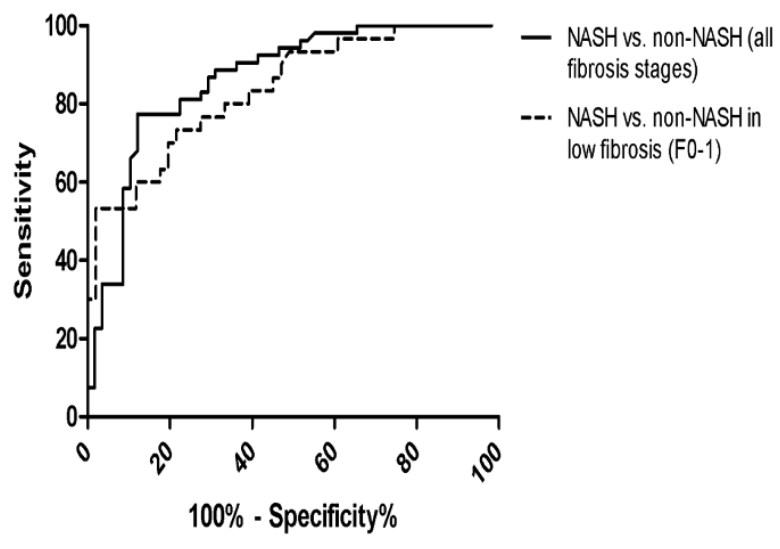

A

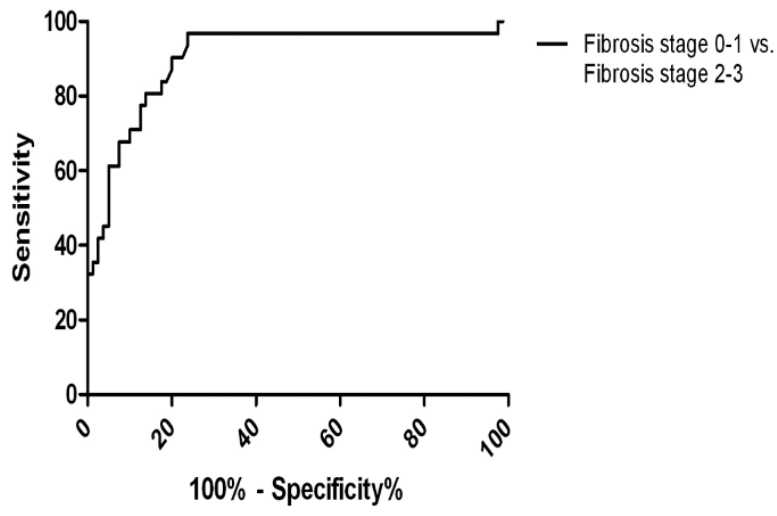

$\mathbf{B}$

Fig. 3. ROC curves for ${ }^{13} \mathrm{C}$-methionine breath test outcome parameter cumulative percentage recovery after 90 min test time $\left(\mathrm{cPDR}_{1.5 \mathrm{~h}}\right.$ ) in patients with non-alcoholic fatty liver (NAFL). A: for differentiation of non-NASH (steatosis and borderlinediagnosis) from NASH in the total cohort (solid line) and a subgroup of patients $(\mathrm{n}=92)$ with mild $(\mathrm{F} 0-1)$ fibrosis (dashed line). B: for differentiation of non-significant (F0-1) from advanced (F2-3) fibrosis in 99 patients with borderline diagnosis or definite NASH.

A cPDR $_{1.5 \mathrm{~h}}$ of $<4.20 \%$ was chosen as an acceptable cut-off value to separate patients with NASH from non-NASH in the total cohort (sensitivity: $81 \%$; specificity: $76 \%$ ). In the subgroup of low fibrosis (F0-1), the optimal cut-off value increased to $4.6 \%$, but the

Table 2. Multiple regression analysis of the relationship between breath test outcome $\left(\mathrm{cPDR}_{1.5 \mathrm{~h}}\right)$ and histological and biochemical parameters.

\begin{tabular}{|c|c|c|c|c|}
\hline & $\begin{array}{c}\text { Regression } \\
\text { coefficient }\end{array}$ & $\begin{array}{l}\text { Standardized } \\
\text { regression } \\
\text { coefficient }\end{array}$ & $\begin{array}{l}\text { Standard } \\
\text { error }\end{array}$ & p-Value \\
\hline Intercept & 6.219 & - & 0.622 & $<0.001$ \\
\hline NASH activity score & -0.449 & -0.448 & 0.054 & $<0.001$ \\
\hline Stage of fibrosis & -0.732 & -0511 & 0.075 & $<0.001$ \\
\hline BMI & -0.023 & -0.074 & 0.016 & 0.156 \\
\hline Cholesterol & 0.006 & 0.180 & 0.002 & $<0.001$ \\
\hline Triglycerides & 0.000 & -0.031 & 0.001 & 0.535 \\
\hline ALT & -0.001 & -0.025 & 0.002 & 0.622 \\
\hline Diabetes & -0.087 & -0.041 & 0.108 & 0.420 \\
\hline
\end{tabular}

Corrected R $2=0.76$ for the entire model

Abbreviations: ALT: alanine aminotransferase; BMI: body mass index. 
overall test performance declined slightly (sensitivity $77 \%$; specificity $73 \%$ ).

In a similar approach, the accuracy of MeBT to differentiate advanced (F2-3) from mild (F0-1) fibrosis within the NAFLD cohort was estimated (Fig. 3b). The area under the curve (AUROC) was 0.90. A cPDR value $<3.65 \%$ was calculated to be the cut-off value with an optimal balance between sensitivity and specificity.

\section{DISCUSSION}

In the present study, we demonstrated that non-alcoholic steatohepatitis is associated with a substantial impairment of hepatic mitochondrial function, which can be measured in vivo by a simple non-invasive ${ }^{13} \mathrm{C}$ breath test. We found a significant and independent inverse correlation of ${ }^{13} \mathrm{C}$-methionine excretion with $\mathrm{NASH}$ activity, measured by the NASH scoring system and fibrosis stage. The rising prevalence of NAFLD which shows a progressive disease in approximately $10-20 \%$ of patients, and the known disadvantages of liver biopsy (invasiveness, potentially fatal complications and sampling error) illustrate the need for new non-invasive diagnostic techniques. Ideally, these diagnostics should discriminate simple steatosis from steatohepatitis, and differentiate initial and advantage stages of fibrosis. Most promising progress has been made in developing non-invasive techniques for the quantification of fibrosis and inflammation. Yoneda et al. evaluated the performance of transient elastography in 97 NAFLD patients and reported a $88 \%$ sensitivity and $74 \%$ specificity for diagnosis of septal fibrosis $(F \geq 2)$ corresponding with an area under the receiving operating curve (AUROC) of 0.87 [20]. More recently, cytokeratin $18(\mathrm{CK}-18)$ has been introduced as a novel, promising serum parameter for non-invasive diagnosis of NASH. CK-18 reflects the amount of liver cell apoptosis, which is characteristic for disease progression from simple steatosis to steatohepatitis. The accuracy (AUROC) of CK-18 to differentiate NAFL from NASH ranges from 0.83 to 0.88 , depending on the study setting [21-23].

Analogous to liver cell apoptosis, mitochondrial dysfunction is a key characteristic of non-alcoholic fatty liver that promotes the development of steatosis and further progression to steatohepatitis: inherited defects of beta oxidation and pharmacological inhibition of mitochondrial respiratory chain activity in rodents are associated with steatohepatitis that is histologically indistinguishable from NAFLD in humans [6, 7]. Many genes encoding mitochondrial proteins in skeletal muscle and fat are negatively correlated with body mass $[8,24,25]$ NAFLD is commonly associated with obesity and insulin resistance; both conditions are characterized by decreased oxygen consumption, ATP production, and reduced content of respiratory proteins in the fat, muscle and liver [8]. Although the metabolic pathway of methionine within hepatocytes does not involve enzymes of beta oxidation or respiratory chain complex, it seems to be very sensitive to conditions of mitochondrial stress, as we could demonstrate in previous studies of HIV/HCV and antiviral drug related mitochondrial toxicity.[15, 26]
Spahr et al. used this technique first for the examination of selected patients with severe $(>40 \%)$ steatosis and reported a significant decay $(-49 \%$ compared to healthy controls) of mitochondrial methionine decarboxylation in this patient group [12]. To our knowledge, our study is the first evaluating the diagnostic power of a new non-invasive metabolic function test in a large cohort of metabolically well characterized patients. We could demonstrate as a major finding that individual ${ }^{13} \mathrm{C}$-methionine breath test performance is associated with histological parameters that are characteristic for non-alcoholic steatohepatitis. Including all stages of fibrosis, the accuracy of the ${ }^{13} \mathrm{C}$-methionine breath test, to separate patients with steatohepatitis from patients with a pure steatosis, seems to be comparable to the diagnostic performance of serum CK18 measurements. These promising results, however, have to be interpreted cautiously since further analyses correcting for fibrosis showed a decline in accuracy for this relevant diagnostic question (AUROC 83\%). Whether this reduction in diagnostic accuracy was caused by the weak, but positive, correlation of key histopathological features, NASH activity and fibrosis $(\mathrm{r}=0.36, \mathrm{p}<0.001)$, or by the relatively small number of patients remains unclear and should be addressed in further studies. In an analogous fashion, the validity of the MeBT as a non-invasive marker of bepatic fibrosis cannot be conclusively judged from this study, given the fact that the limited number of patients with advanced fibrosis also had higher grades of NASH activity, a factor that may independently, but concordantly, influence the outcomes of the breath test. Thus, in order to gain a better understanding regarding sensitivity and specificity of this non-invasive technique, it would be necessary to examine larger groups of patients at well defined stages of NASH activity and fibrosis. Ideally, such studies should also implement other non-invasive markers such as CK-18 for a better group stratification, which was not available during the time this study was conducted.

Furthermore, the pathophysiological basis of the ${ }^{13} \mathrm{C}$-methionine breath test has to be clarified more in detail. At this point, the underlying molecular mechanism that determines the poor breath test results in patients with NAFL, as well as in other mitochondriaeffecting liver diseases such as hepatitis C or HIV, are largely unclear. Owing to the pilot nature of this study, the small amounts of liver tissue available from the patients has been primarily used for histological examinations. Nevertheless, more detailed analyses of mitochondria-specific structures and functions are certainly warranted in order to establish the ${ }^{13} \mathrm{C}$-methionine breath test as a routine instrument in clinical practice. Along these lines, a recently published study by Mawatari et al. examined mitochondrial beta-oxidation in patients with NASH using a non-invasive ${ }^{13} \mathrm{C}$-octanoate breath test [27]. In this study, no significant differences in breath test performance between the study groups (NAFL, NASH) were found, suggesting that the beta-oxidation is not generally impaired in patients with non-alcoholic steatohepatitis.

With respect to the limitations stated above, what could the potential applications and advantages for the $\mathrm{MeBT}$ in clinical practice be? We propose the two fol- 
lowing scenarios: in patients who have suspected NAFLD, a cumulative ${ }^{13} \mathrm{C}$-exhalation $\left(\mathrm{cPDR}_{1.5 \mathrm{~h}}\right)$ $<4.2 \%$ indicates definite NASH, and should be interpreted in favour of performing a liver biopsy. In those patients with a histologically confirmed diagnosis, the MeBT might a useful tool to prospectively monitor disease progression or potential benefits due to therapeutic interventions, as could be shown recently by our group [16, 27]. The majority of NASH patients present at early stages of fibrosis at the time of primary diagnosis. These patients are typically not at risk of rapid fibrosis progression, and might experience a greater benefit from non-invasive diagnostic procedures which accurately measure the extent of NASH activity, rather than liver fibrosis.

Given the lack of specificity for inflammatory and fibrotic changes, and in consideration of the impact of NAFLD-independent factors (alcohol, viral infections, etc.), it seems unlikely that the ${ }^{13} \mathrm{C}$-methionine breath test - as with other non-invasive markers - could completely replace liver biopsies for diagnosing and staging of NASH. Nevertheless, the ${ }^{13} \mathrm{C}$-methionine breath test could serve as a valuable supporting diagnostic instrument in NAFLD and NASH, which may help to individualize diagnostic procedures, and to minimize the necessity of liver biopsies in this chronic disease.

Statement of Interests: Authors' declaration of personal interests: none

Declaration of funding interests: None

\section{REFERENCES}

1. McCullough AJ. The clinical features, diagnosis and natural history of nonalcoholic fatty liver disease. Clin Liver Dis 2004;8(3):521-33, viii.

2. Clark JM. The epidemiology of nonalcoholic fatty liver disease in adults. J Clin Gastroenterol 2006;40(3 Suppl 1):S5-10.

3. Farrell GC, Larter CZ. Nonalcoholic fatty liver disease: from steatosis to cirrhosis. Hepatology 2006;43(2 Suppl 1):S99-S112.

4. Begriche K, Igoudjil A, Pessayre D, Fromenty B. Mitochondrial dysfunction in NASH: causes, consequences and possible means to prevent it. Mitochondrion 2006; 6(1):1-28.

5. Caldwell SH, Swerdlow RH, Khan EM, Iezzoni JC, Hespenheide EE, Parks JK, et al. Mitochondrial abnormalities in non-alcoholic steatohepatitis. J Hepatol 1999;31(3): 430-4.

6. Perez-Carreras M, Del Hoyo P, Martin MA, Rubio JC, Martin A, Castellano G, et al. Defective hepatic mitochondrial respiratory chain in patients with nonalcoholic steatohepatitis. Hepatology 2003;38(4):999-1007.

7. Ibdah JA, Perlegas P, Zhao Y, Angdisen J, Borgerink H, Shadoan MK, et al. Mice heterozygous for a defect in mitochondrial trifunctional protein develop hepatic steatosis and insulin resistance. Gastroenterology 2005;128(5): 1381-90.

8. Valerio A, Cardile A, Cozzi V, Bracale R, Tedesco L, Pisconti A, et al. TNF-alpha downregulates eNOS expression and mitochondrial biogenesis in fat and muscle of obese rodents. J Clin Invest 2006;116(10):2791-8.

9. Wei Y, Rector RS, Thyfault JP, Ibdah JA. Nonalcoholic fatty liver disease and mitochondrial dysfunction. World J Gastroenterol 2008;14(2):193-9.
10. Milazzo L, Piazza M, Sangaletti O, Gatti N, Cappelletti A, Adorni F, et al. $\left[{ }^{13} \mathrm{C}\right]$ Methionine breath test: a novel method to detect antiretroviral drug-related mitochondrial toxicity. J Antimicrob Chemother 2005;55(1):84-9.

11. Armuzzi A, Marcoccia S, Zocco MA, De Lorenzo A, Grieco A, Tondi P, et al. Non-Invasive assessment of human hepatic mitochondrial function through the ${ }^{13} \mathrm{C}-\mathrm{me}-$ thionine breath test. Scand J Gastroenterol 2000;35(6): 650-3.

12. Spahr L, Negro F, Leandro G, Marinescu O, Goodman KJ, Rubbia-Brandt L, et al. Impaired hepatic mitochondrial oxidation using the ${ }^{13} \mathrm{C}$-methionine breath test in patients with macrovesicular steatosis and patients with cirrhosis. Med Sci Monit 2003;9(1):CR6-11.

13. Banasch M, Goetze O, Hollborn I, Hochdorfer B, Bulut $\mathrm{K}$, Schlottmann $\mathrm{R}$, et al. ${ }^{13} \mathrm{C}$-methionine breath test detects distinct hepatic mitochondrial dysfunction in HIVinfected patients with normal serum lactate. J Acquir Immune Defic Syndr 2005;40(2):149-54.

14. Banasch M, Goetze O, Knyhala K, Potthoff A, Schlottmann R, Kwiatek MA, et al. Uridine supplementation enhances hepatic mitochondrial function in thymidine-analogue treated HIV-infected patients. Aids 2006; 20(11):1554-6.

15. Banasch M, Emminghaus R, Ellrichmann M, Schmidt WE, Goetze O. Longitudinal effects of hepatitis $\mathrm{C}$ virus treatment on hepatic mitochondrial dysfunction assessed by $\mathrm{C}$-methionine breath test. Aliment Pharmacol Ther 2008;28(4):443-9.

16. Banasch M, Goetze O, Schmidt WE, Meier JJ. Rimonabant as a novel therapeutic option for nonalcoholic steatohepatitis. Liver Int 2007;27(8):1152-5.

17. Portincasa P, Grattagliano I, Lauterburg BH, Palmieri VO, Palasciano G, Stellaard F. Liver breath tests non-invasively predict higher stages of non-alcoholic steatohepatitis. Clin Sci (Lond) 2006;111(2):135-43.

18. Schmilovitz-Weiss H, Niv Y, Pappo O, Halpern M, Sulkes J, Braun $\mathrm{M}$, et al. The ${ }^{13} \mathrm{C}$-Caffeine Breath Test Detects Significant Fibrosis in Patients With Nonalcoholic Steatohepatitis. J Clin Gastroenterol 2008;42(4):408412.

19. Kleiner DE, Brunt EM, Van Natta M, Behling C, Contos MJ, Cummings OW, et al. Design and validation of a histological scoring system for nonalcoholic fatty liver disease. Hepatology 2005;41(6):1313-21.

20. Yoneda M, Mawatari H, Fujita K, Endo H, Iida H, Nozaki $\mathrm{Y}$, et al. Noninvasive assessment of liver fibrosis by measurement of stiffness in patients with nonalcoholic fatty liver disease (NAFLD). Dig Liver Dis 2008;40(5): 371-8.

21. Wieckowska A, Zein NN, Yerian LM, Lopez AR, McCullough AJ, Feldstein AE. In vivo assessment of liver cell apoptosis as a novel biomarker of disease severity in nonalcoholic fatty liver disease. Hepatology 2006;44(1):27-33.

22. Feldstein AE, Wieckowska A, Lopez AR, Liu YC, Zein NN, McCullough AJ. Cytokeratin-18 fragment levels as noninvasive biomarkers for nonalcoholic steatohepatitis: a multicenter validation study. Hepatology 2009;50(4): 1072-8.

23. Diab DL, Yerian L, Schauer P, Kashyap SR, Lopez R, Hazen SL, et al. Cytokeratin 18 fragment levels as a noninvasive biomarker for nonalcoholic steatohepatitis in bariatric surgery patients. Clin Gastroenterol Hepatol 2008;6(11):1249-54.

24. Sparks LM, Xie H, Koza RA, Mynatt R, Hulver MW, Bray GA, et al. A high-fat diet coordinately downregulates genes required for mitochondrial oxidative phosphorylation in skeletal muscle. Diabetes 2005;54(7):1926-33.

25. Patti ME, Butte AJ, Crunkhorn S, Cusi K, Berria R, Kashyap S, et al. Coordinated reduction of genes of oxidative metabolism in humans with insulin resistance and 
diabetes: Potential role of PGC1 and NRF1. Proc Natl Acad Sci U S A 2003;100(14):8466-71.

26. Banasch M, Knyhala K, Kollar S, Serova K, Potthoff A, Schlottmann R, et al. Disease- and treatment-related predictors of hepatic mitochondrial dysfunction in chronic HIV infection assessed by non-invasive (13)C-methionine breath test diagnostic. Eur J Med Res 2008;13(9):401-8.

27. Banasch M, Frank J, Serova K, Knyhala K, Kollar S, Potthoff $\mathrm{A}$, et al. Impact of antiretroviral treatment on (13)C-methionine metabolism as a marker of hepatic mitochondrial function: a longitudinal study. HIV Med. 2010 May 23. [Epub ahead of print]
Received: July 28, 2010/ Accepted: September 30, 2010

Address for correspondence:

Dr. Matthias Banasch

Department of Medicine 1

St. Josef-Hospital

University of Bochum

Gudrunstrasse 56

44791 Bochum

Germany

Fax: +492345093586

Email: mbanasch@gmx.de 